\title{
Delitos contra la libertad sexual
}

\section{Crimes against sexual freedom}

\section{RESUMEN}

Los delitos contra la libertad e indemnidad sexual son hechos producidos a diario en nuestro país y son expresión de una sociedad decadente en valores; son, a la vez, fenómenos de alarma social, debido a que los medios de comunicación los enfocan como un elemento de la problemática social. La violencia sexual es un problema de salud pública en nuestro país y muchos otros. Existen múltiples definiciones para los términos: violencia, violación, abuso o delito contra la libertad sexual, todos referidos a este acto, en el que básicamente se afecta la voluntad y libertad sexual de la víctima. Finalmente, al constituirse estos actos en delitos, también deben tener sanciones propias del mismo, todas dependientes de procesos jurídico-penales.

Palabras clave: libertad; violación; crimen; víctimas de crimen; violencia sexual

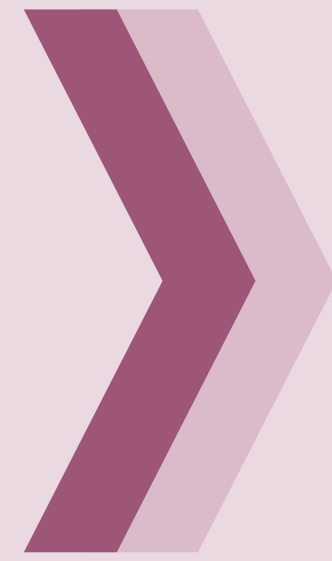

\section{Ulises Papillón Mejía-Rodríguez, José Yamid Bolaños-Cardozo, ${ }^{2}$ Alex Mejía-Rodríguez ${ }^{3}$}

1 Médico legista. Máster en Medicina forense. Doctorando en Criminalística. División Médico Legal I de Juli, Chucuito, Puno, Perú.

2 Médico legista. Máster en Calidad y Gestión Integral. Doctorando en Criminalística. Docente en Ecisalud, Tunja, Boyacá, Colombia.

3 Interno de Medicina. EsSalud, Lima. Universidad Nacional Jorge Basadre Grohmann, Tacna, Perú.

\begin{abstract}
Crimes against sexual freedom and indemnity are events occurring daily in our country and that are a reality, are both phenomena of social alarm, because the media's focus as an element of social problems. Sexual violence is a public health problem in our country and many others. There are multiple definitions of the terms: violence, rape, abuse or crime against sexual freedom, all referred to this act, which basically will and sexual freedom of the victim is affected. Finally when these acts constitute offenses, they should also have own sanctions thereof, all dependent criminal legal process.
\end{abstract}

KEY wORDs: freedom; rape; crime; crime victims; sexual violence

\section{INTRODUCCIÓN}

En todo el Perú, a diario se producen delitos contra la libertad e indemnidad sexual contra mujeres mayores y menores de edad, pero también contra menores de sexo masculino, que quedan afectados de por vida, tanto psicológica como físicamente, como consecuencia de esta conducta reprochable por las sociedades civilizadas. Este comportamiento genera gran alarma social, pues a diario nos informarnos mediante los medios de comunicación de hechos que se cometen en agravio de mujeres y menores de edad que, a pesar de los esfuerzos de los grupos feministas (Manuela Ramos y Flora Tristán) por la defensa de los derechos de la mujer y el niño, así como del endurecimiento de las penas por parte de los órganos administradores 
de justicia, se sigue observando índices alarmantes de abusos sexuales. Las sanciones graves para los agresores sexuales no solucionará el problema de fondo, debido a la falta de claridad y precisión en algunos tipos penales y por la falta de profundización de las investigaciones, entre otros factores. Es necesario hacer cambios profundos en la legislación peruana, de manera que se brinden garantías constitucionales, tanto a las víctimas como a los agresores.

\section{VIOLENCIA SEXUAL}

La violencia sexual es un problema relevante de salud pública en nuestro país. No resulta fácil encontrar una definición que sea lo suficientemente abarcadora y operativamente útil, debido a la complejidad de su etiología como por la variedad de sus formas de manifestarse. Se trata de conductas cuyo juicio social negativo depende de aspectos culturales y de valores hegemónicos en cada momento y lugar.

La violencia sexual, desde el punto de vista desde la salud pública, es un fenómeno general de la violencia, que la Organización Mundial de la Salud (OMS) lo define como: "El uso intencional de la fuerza o el poder físico, de hecho o como amenaza, contra uno mismo, otra persona o un grupo, que cause lesiones, muerte, daños psicológicos, trastornos del desarrollo o privaciones".

La violencia sexual presenta una manifestación muy específica, pero no aislada de las otras formas de violencia, donde hacen sinergismo las distintas naturalezas de violencia, como la violencia física, la violencia psíquica, las privaciones o el descuido $y$, sobre todo, la muy conocida violencia sexual. Así es que podemos diferenciar, dentro de la violencia interpersonal, la que se ocasiona por la familia, la pareja o la comunidad en menores, en la pareja o en adultos mayores, entre personas conocidas como entre personas extrañas, así como también una violencia sexual colectiva, que podría ser social, política y económica. ${ }^{1-3}$ Por esto, no dudamos en decir que la violencia sexual puede ser un fenómeno familiar o comunitario, pero también un problema de naturaleza colectiva. ${ }^{4}$

Es así que la OMS y la Organización Panamericana de la Salud (OPS) definen a la violencia sexual como todo acto sexual, la tentativa de consumar un acto sexual, los comentarios o insinuaciones sexuales no deseados, o las acciones para comercializar o utilizar, de cualquier otro modo, la sexualidad de una persona, mediante coacción por otra persona, independientemente de la relación de esta con la víctima, en cualquier ámbito, incluidos el hogar y el lugar de trabajo. Agrega que la coacción puede abarcar una amplia gama de grados de uso de la fuerza. Además de la fuerza física, puede entrañar la intimidación psíquica, la extorsión u otras amenazas, como la de daño físico, despedir a la víctima del trabajo o de impedirle obtener el trabajo que busca. También puede ocurrir cuando la persona agredida no está en condiciones de dar su consentimiento, por ejemplo, porque está ebria, bajo los efectos de un estupefaciente o dormida, o es mentalmente incapaz de comprender la situación.

Según el actus reus del crimen de violación, bajo el derecho internacional, está constituido por la penetración sexual sin el consentimiento de la víctima, aunque sea leve, de la vagina o el ano, o cualquier objeto utilizado, o la boca de la víctima por el pene del perpetrador. En 2009, en una muestra nacional representativa de 1242 niñas y mujeres de 13 a 24 años de edad en Suazilandia (en África Austral), se encontró que 33,2\% de las entrevistadas informaron haber sufrido algún incidente de violencia sexual antes de los 18 años de edad. ${ }^{5}$

El Estatuto de la Corte incorporó en la definición de violación un término mucho más amplio que el de penetración: la "invasión", para que resultara neutro en cuanto al sexo. La definición de invasión incluye no solo la penetración de un órgano sexual, sino también cualquier tipo de abuso sexual con objetos o con partes del cuerpo. ${ }^{6-10}$

\section{ANTECEDENTES HISTÓRICOS}

La violación, entendida como acceso carnal, ha sido contemplada por las legislaciones antiguas:11-14

En Roma se castigaba con la pena de muerte a quienes ejercían violencia sobre personas casadas o solteras. En el Código de Hammurabi de los babilónicos y caldeos se sancionaba de una manera enérgica; la agraviada, la sociedad y 
todos los dioses eran las víctimas. La sanción que se aplicaba al violador era la pena de muerte por ahorcamiento. El derecho hebreo tenía penas más drásticas: se sancionaba con la pena de muerte al violador, así como a los familiares más cercanos.

El derecho canónico también sancionaba este delito con la pena de muerte, pero tenía como requisito que la víctima sea virgen y sea desflorada; si esta no reunía esas características, no se consideraba como tal y se sancionaba con penas más leves. En las leyes españolas, el Fuero Juzgo castigaba al hombre libre con cien azotes y al siervo a morir quemado en fuego. El Fuero Viejo de Castilla determinaba la muerte de quien forzaba a una mujer virgen o no. Las partidas amenazaban con la pena de muerte al hombre que robara a una mujer viuda de buena fama, virgen, casada o religiosa, o viviere con algunas de ellas por la fuerza.

En el Perú, los incas sancionaban al violador con expulsión del pueblo; solo se aplicaba la pena de muerte a los reincidentes. En la época de la Colonia, la cifra negra de la criminalidad aumentó debido al abuso y flagelo de los cuales eran víctimas nuestros indígenas. En la época de la República, estando vigente el Código de 1924, había pena de muerte para el violador que agraviaba a menores de siete años; luego fue sustituida por la pena de internamiento. Posteriormente, en la Constitución de 1979 y en la actual, solo se aplica la pena de muerte en caso de traición a la Patria en situación de guerra exterior.

\section{DELITOS SEXUALES}

Al hablar de delitos sexuales, estamos aludiendo una categoría jurídico-penal. Se trata de actividades sexuales o vinculadas con lo sexual y que constituyen delito en tanto constituyan acciones $u$ omisiones típicas, o lo que se prevé como delitos en una ley penal. En los delitos sexuales, el bien jurídico protegido por el derecho es la libertad sexual de las personas o bien la moralidad pública. ${ }^{15}$

Desde una óptica médico-forense, los delitos que presentan mayor interés son aquellos que afectan la libertad sexual y que presentan una característica común en nuestra legislación peruana, que es la existencia de alguna clase de actividad sexual cumplida bajo alguna forma de violencia o de amenaza. ${ }^{16-18}$

La actividad sexual puede ser el coito o alguna otra forma de gratificación sexual por parte del agresor. Si la actividad sexual consiste en el coito, las legislaciones suelen denominar a este delito como violación, mientras que en la ley brasileña es estupro, que se refiere, en general, al acceso carnal o conjunción carnal. Los distintos países definen al coito de forma similar y se ajustan a lo que establezca la ley a través de su código penal, según sea el caso. Mientras en algunos países solo podrá haber violación por penetración vaginal del pene en erección, en otros se incluye la penetración rectal y en otros, además, la bucal. Algunas legislaciones también tipifican la violación en los casos de introducción de partes del cuerpo distintas del pene e incluso de cuerpos extraños. ${ }^{19}$

La actividad sexual distinta del coito se llama, en los diferentes códigos, actos contra el pudor, abuso deshonesto o atentado violento al pudor. La definimos por exclusión, como cualquier forma de gratificación sexual distinta del coito, o lo que sería su equivalente a un acto sexual que no encaja en la tipificación amplia de la violación sexual..20

La violencia sexual presenta, por definición legal o jurídica, el uso de la violencia como un medio útil para practicar la actividad sexual, que vendría a ser el segundo elemento sustancial en los delitos contra la libertad sexual. ${ }^{21-23}$ La violencia consiste en el uso de la fuerza física, como también el uso de la amenaza como medio de su empleo, o el abuso de alguna situación de superioridad. Casi todas las legislaciones, o la gran mayoría, reconocen una serie de circunstancias en que la violencia se presume; estos son los supuestos de delitos sexuales ope legis, es decir, por disposición de la ley.

\section{CONCLUSIONES}

Los delitos contra la libertad e indemnidad sexual son hechos producidos a diario en nuestro país; son fenómenos que ocurren en nuestro medio social debido a debilidades en el sistema de legislaciones.

Existen múltiples definiciones para los términos violación, violencia, delito contra la libertad 
sexual y otros; todas tienen como punto común la irrupción a la libertad sexual de las personas afectadas.

Deben realizarse profundos cambios en las legislaciones de nuestro país, modificaciones de leyes para sancionar este tipo de delitos. Únicamente haciendo revisión de estas leyes y tomando posteriormente medidas de control más estrictas se podrá disminuir la problemática de estos delitos.

\section{REFERENCIAS BIBLIOGRÁFICAS}

I. García-Moreno C, Jansen H, Watts C, Ellsberg M, Heise L; Organización Mundial de la Salud (OMS). Estudio multipaís de la OMS sobre salud de la mujer y violencia doméstica contra la mujer [Internet]. Ginebra, Suiza: OMS; 2005 [citado I8 may 2015]. Disponible en: http://www.who.int/gender/ violence/who multicountry_study/summary_report/ summaryreportSpanishlow.pdf

2. Heise L, Ellsberg M, Gottemoeller M; Center for Health and Gender Equity. Ending violence against women. Population Reports, Series L, N ${ }^{\circ}$ II [Internet]. Baltimore (Maryland), EE. UU.: Johns Hopkins University School of Public Health, Population Information Program; 1999 [citado 25 may 2015]. Disponible en: https://www.k4health.org/sites/default/files/L\%20I I.pdf

3. Tjaden P, Thoennes N; National Institute of Justice, National Center for Injury Prevention and Control. Full report of the prevalence, incidence, and consequences of violence against women: findings from the National Violence Against Women Survey [Internet]. Washington, DC, EE. UU.: U. S. National Institute of Justice; 2000 [citado 23 may 20I5]. Disponible en: https://www.ncjrs.gov/pdffiles I/nij//8378I.pdf

4. Jewkes R, Sikweyiya Y, Morrell R, Dunkle K. Gender inequitable masculinity and sexual entitlement in rape perpetration South Africa: findings of a cross-sectional study. PLoS One. 20I I;6(I2):e29590.

5. Reza A, Breiding MJ, Gulaid J, Mercy JA, Blanton C, Mthethwa $Z$, Bamrah S, et al. Sexual violence and its health consequences for female children in Swaziland: a cluster survey study. Lancet. 2009;373(9679):1966-72.

6. Di Maio JM, Dana SE. Homicidio relacionado con el sexo. En: Di Maio,Vincent JM, editores. Manual de patología forense. Madrid, España: Díaz de Santos; 2003. p. 165-7.

7. Gayet J. Manual de la policía científica. Madrid, España: Zeus; 1965.
8. Calabuig G, Villanueva E. Delitos contra la libertad sexual. En: Calabuig G,Villanueva E, editores. Medicina legal y toxicología. $5^{\mathrm{a}}$ ed. Barcelona, España: Masson; 1998: 495-5I4.

9. Guzmán C. Manual de criminalística. Buenos Aires, Argentina: La Roca; 2000. p. 487.

10. Knight B. Delitos sexuales. En: Knight B, editor. Medicina forense de Simpson. México, D. F.: El Manual Moderno; 1994. p. 231-45.

I I. Kvitko LA. La violación: peritación medicolegal en las presuntas víctimas del delito. $2^{\mathrm{a}}$ ed. México, D. F.:Trillas; 1995.

12. Vanrell P.Violencia sexual. En:Vanrell P, editor. Sexología forense. Montes Claros (Minas Gerais), Brasil:Unimontes; 200I.p. 29I-394.

13. Rodríguez $H$. Abuso sexual en niños: enfoque médico-legal. En: Baráibar R, editor. La salud en la infancia y la adolescencia. Montevideo, Uruguay: Unimontes; 1999. p. 141-9.

14. Simonin C. Medicina legal judicial. Barcelona, España: Jims; 1962.

15. Policía Nacional del Perú. Manual de criminalística. Lima, Perú: JMD; 2006. p. 232.

16. Grossin C, Sibille I, Lorin de la Grandmaison G, Banasr A, Brion F, Durigon M.Analysis of 418 cases of sexual assault. Forensic Sci Int. 2003; | 31 (2-3): 125-30.

17. Gray-Eurom K, Seaberg DC, Wears RL. The prosecution of sexual assault cases: correlation with forensic evidence. Ann Emerg Med. 2002;39(I):39-46.

18. Heger AH,Ticson L, Guerra L, Lister J, Zaragoza T, McConnell G, et al. Appearance of the genitalia in girls selected for nonabuse: review of hymenal morphology and nonspecific findings.J Pediatr Adolesc Gynecol. 2002;15(I):27-35.

19. Glaser D, Frosh S.Abuso sexual de niños. Buenos Aires,Argentina: Paidós; 1997.

20. American Academy of Pediatrics Committee on Child Abuse and Neglect. Guidelines for the evaluation of sexual abuse of children: subject review. Pediatrics. 1999; 103(I):186-91.

21. Burgess AW, Holmstrom LL. Rape trauma syndrome. Am J Psychiatry. 1974; |3I(9):98|-6.

22. República de Colombia. Delitos contra la libertad sexual. En: Código penal colombiano (Ley 599 de 2000). Bogotá, D. C., Colombia: Congreso de Colombia; 2000. p. 184, 190.

23. Zajaczkowski R. Manual de criminalística. Buenos Aires,Argentina: Ciudad Argentina; 1998.

\section{Correspondencia}

Dr. Ulises Mejía-Rodríguez

ulisesmejiarodriguez@hotmail.com

\section{Conflictos de interés}

Los autores declaran no tener conflictos de interés durante el planteamiento, ejecución de la investigación y la elaboración del artículo para su publicación.

Fecha de recepción: I de junio de 2015

Fecha de aceptación: 30 de junio de 2015 